\title{
Audiencias activas y desigualdad de ingresos: análisis exploratorio de la conversación en Twitter
}

\author{
Laura Pérez Altable \\ Universidad Nebrija \\ lauraperezaltable@gmail.com \\ https://orcid.org/0000-0003-3114-6371
}

\author{
Ana Serrano Tellería \\ Universidad de Castilla la Mancha \\ anaserranotelleria@gmail.com \\ https://orcid.org/0000-0003-1625-4411
}

\author{
Ariadna Fernández Planells \\ Universitat Politècnica de València \\ arferpla@upv.es \\ https://orcid.org/0000-0003-4600-9345
}

\section{Actives audiences and income inequality: An exploratory analysis of the conversation on Twitter}

\begin{abstract}
RESUMEN
La desigualdad de ingresos es uno de los mayores desafíos de nuestras sociedades y uno de los temas principales de la agenda política reciente (Pickett y Wilkinson, 2015: 132), especialmente desde el colapso financiero global. Como Mike

Savage (2016) afirma, "la desigualdad está en todas partes, en las representaciones que los medios hacen de la realidad, así como en nuestra vida social diaria o en las redes sociales".

Las redes sociales tienen el potencial de facilitar una democracia más participativa y un valor disruptivo para desafiar los intereses tradicionales y los modos de poder comunicativo (Loader y Mercea, 2011). Por lo tanto, nuestro objetivo es describir cómo se crea

la opinión pública en relación con el concepto de desigualdad de ingresos en Twitter. Desde un punto de vista metodológico, tras haber realizado un análisis cuantitativo en trabajos previos, proponemos explorar el cualitativo a través de la recolección de 102.082 tuits en 2018 sobre el concepto de desigualdad de ingresos.
\end{abstract}

PALABRAS CLAVE

Desigualdad de ingresos; Esfera pública digital; Redes sociales; Twitter

\section{ABSTRACT}

The income inequality is one of the major challenges of our societies and one of the main topics on the recent political agenda (Pickett $\&$ Wilkinson, 2015: 132), especially since the global financial collapse. As Mike Savage (2016) affirms, "inequality is everywhere: in the representations that the media makes of reality, as well as in our daily social life or in social networks".

Paying attention to digitally mediated conversation, the research will focus on Twitter as a digital platform and social network used for set the topic in the digital public sphere. Social media have the potential to facilitate more participative democracy and a disruptive value for challenging traditional interests and modes of communicative power (Loader \& Mercea, 2011). Thus, we focus on identifying how public opinion is created in relation to the concept of income inequality in social networks as Twitter. From a methodological point of view, we propose an analysis of the tweets published by different users on Twitter around the concept of income inequality.

KEYWORDS

Income Inequality; Digital Public Sphere;

Social Networks; Twitter 


\section{Introducción}

La desigualdad de ingresos es uno de los temas públicos más importantes para las sociedades (Song, Price Guvenen, Bloom y Von Watcher, 2019), especialmente durante la última década, con el estallido de una de las mayores crisis financieras y económicas de la historia. Además, en las últimas décadas, la desigualdad de ingresos ha experimentado un aumento significativo, sobre todo en los países desarrollados (Aghion, et al., 2018, p.1). Por ello, acabar con la desigualdad de ingresos se ha convertido en uno de los mayores retos de nuestras sociedades, reflejado en el incremento de publicaciones académicas dentro de este ámbito. Sin embargo, la mayoría de estudios se han llevado a cabo desde disciplinas relacionadas con los análisis económicos, sociológicos y políticos. En este estudio, proponemos abordar el análisis del concepto de desigualdad salarial o de ingresos (income inequality en inglés) desde el ámbito de la comunicación. De acuerdo con algunos autores (ver McCall, 2010, p.47), en torno al año 1987 aparece el concepto de desigualdad salarial como unos de los temas importantes dentro de la investigación en Ciencias Sociales. Si bien, es a partir del año 2008 cuando las investigaciones relacionadas con este fenómeno aumentaron considerablemente. En concreto, podemos citar dos obras de referencia que han situado el concepto de desigualdad salarial en el centro de un debate intenso en los últimos años, llegando a una extensa audiencia, aun tratándose de trabajos especializados en economía. Estos trabajos son: The Spirit Level (Wilkinson y Pickett, 2009) y Le Capital au XXle Siècle (Thomas Picketty, 2013). Precisamente, Pickett y Wilkinson afirmaron que "desde la crisis financiera global, la presencia del concepto de desigualdad ha aumentado rápidamente dentro de la agenda política" (Pickett y Wilkinson, 2015, p.132).

En este sentido, en trabajos anteriores, se ha detectado que el concepto de desigualdad de ingresos está en la agenda política, tal y como afirman Pickett y Wilkinson, pero ni los políticos ni los medios de comunicación están introduciendo el concepto en la agenda política y mediática en la esfera pública digital (Díaz-Noci y Pérez-Altable, 2018, p. 87, Pérez-Altable, Fernández-Planells y Serrano-Tellería, 2019). Es por ello que, en este trabajo, nos centraremos en analizar cómo es la conversación en torno al concepto de desigualdad de ingresos que tiene lugar en las plataformas digitales con capacidad de apoyar la formación de una esfera pública en red (Colleoni, Rozza, y Arvidsson, 2014). Específicamente, este trabajo se focaliza en Twitter, dónde realizamos un análisis de contenido sobre los tuits publicados en Twitter durante el año 2018 en relación con el concepto de desigualdad salarial. Dar respuesta a este objetivo general supone responder a: i) qué tipo de contenido se comparte cuando se habla de desigualdad en Twitter; ii) cuáles son las fuentes utilizadas y compartidas; iii) quiénes son los protagonistas o a quién se menciona en los tuits que hablan sobre desigualdad (políticos, organismos, ONG, ciudadanos, etc.); iv) cuál es el carácter de la desigualdad de la que se habla (micro/ macro) y v) cuál es el tema principal de los mensajes sobre desigualdad en Twitter.

Teniendo en cuenta que nuestro trabajo se centra en la conversación en Twitter en torno al concepto de desigualdad, conviene destacar que para la confección de la muestra de este estudio hemos realizado la búsqueda de tuits utilizando las palabras clave "income inequality" en inglés, como veremos más adelante. De acuerdo con esto, los resultados que se presentan en este estudio están vinculados al mundo anglosajón, con especial presencia de tuits originados en Estados Unidos y Reino Unido. Esta delimitación de las palabras clave no permiten generalizar y extrapolar el análisis a otros contextos diferentes, ya que los datos que conforman la muestra responden a una realidad específica.

Este estudio forma parte de un proyecto de investigación en curso llamado "News, Networks and Users in the Hybrid Media System". Dentro de este marco, nuestro objetivo es examinar el papel que desempeñan las audiencias activas en el establecimiento de la agenda pública en el sistema híbrido de medios y que llevamos a cabo en el subproyecto "Transformation of News and Media Industry in the Post-Industrial Era". El presente trabajo es una primera aproximación que nos permite testear la herramienta diseñada así como ofrecer resultados de carácter exploratorio y preliminar que, aunque no pueden ser extrapolados a otros contextos y han de ser interpretados con cautela, aportan una visión lo suficientemente amplia (de un año) para poder ofrecer conclusiones relevantes en respuesta a nuestro objectivo de estudio. Y que podrán ser ampliados en futuras investigaciones durante una etapa más avanzada del proyecto de investigación.

\section{Estado de la cuestión}

Este apartado teórico se divide en dos subapartados, en el primer de ellos se profundiza en el concepto de desigualdad de ingresos y en sus diferentes aproximaciones que nos han permitido definir, en el apartado metodológico, nuestra herramienta de análisis. En el segundo subapartado, se debate la capacidad de las audiencias 
activas y de las plataformas digitales para apoyar la formación de una esfera pública en red.

\subsection{El concepto de desigualdad de ingresos}

Cuando hablamos de desigualdad de ingresos nos referimos a la disparidad que existen en las distribuciones de los ingresos de los individuos, generalmente con una alta concentración de los ingresos en manos de un pequeño porcentaje de la población. En el momento en que esto ocurre, existe una gran brecha entre la riqueza de un segmento de la población en comparación con otro. Es decir, se produce cuando los recursos económicos, en lugar de distribuirse de manera equitativa, se concentran en pocas manos. El ingreso de un individuo se determina por el flujo de dinero que entra en su hogar, después de pagar los impuestos (Keeley, 2018, p. 25). Las entradas de dinero pueden incluir sueldos o salarios, ganancias por inversiones y renta de propiedades, así como cualquier tipo de prestación recibida por parte del Estado. Por último, es importante aclarar que la desigualdad de ingresos está estrechamente vinculada a otros factores de desigualdad (género, etnia o procedencia, entre otros), aunque en este estudio nos centraremos solo en analizar el concepto de desigualdad de ingresos por una cuestión operativa.

Lo primero que cabría destacar es que el concepto de desigualdad de ingresos tiene lugar tanto dentro de una determinada comunidad como entre diferentes comunidades, entendiendo comunidad como el conjunto de personas que tienen ciertos elementos en común. Es decir, es importante el encuadre específico desde el cual se aborda la desigualdad analizada. De esta forma, nos encontramos con dos perspectivas diferentes y específicas, una perspectiva micro, mediante la cual se analizan las desigualdades de las personas en relación con el conjunto de personas que forman una comunidad determinada, y una perspectiva macro, la cual hace referencia a las diferencias entre comunidades.

Dentro del ámbito de la investigación en Ciencias Sociales resulta más frecuente encontrarse con estudios que tratan de analizar la desigualdad entre diferentes comunidades, es decir, utilizando una perspectiva de análisis macro. A este tipo de desigualdad se le ha dado diferentes nombres: World Income Inequality, International Inequality o Cross-national Inequality. También ha sido referida como "La Gran Divergencia" (The Great Divergence). Este concepto significa, a grosso modo, desigualdad entre naciones y países. También podemos encontrar otros términos relacionados para referirnos a este fenómeno: globalización, riqueza de las naciones o pobreza.

La perspectiva micro, a la que nos hemos referido en el párrafo está relacionada con la riqueza de una persona individual cuando es comparada con la riqueza de otras personas dentro de una comunidad dada, siendo esta comunidad una nación determinada o una ciudad, por ejemplo. Ha sido definida como la variación de los niveles de vida en toda una población (McKay, 2002). De alguna manera, la desigualdad individual o doméstica está relacionada con la clase social ya que, de acuerdo con Debraj Ray, la desigualdad salarial "es la disparidad fundamental que permite a un individuo ciertas elecciones materiales, mientras que niega a otro individuo realizar esas mismas elecciones" (Ray, 199, p. 170). Algunas expresiones relacionadas son: desigualdad de salario (calculada en relación con los salarios que un empleador paga o que un asalariado recibe, pero no en relación a las rentas individuales), pobreza, impuestos, y clase social. Así mismo, debemos aclarar que, aunque en un principio pudiera parecer que la pobreza es lo mismo que desigualdad de ingresos, son conceptos diferentes y la pobreza debe ser considerada como una consecuencia de esta (McKay, 2002; Pickett y Wilkinson, 2015, p. 132). La noción de pobreza se basa en conceptos que son fácilmente medibles, por ejemplo, rentas, impuestos o beneficios. En este sentido, podemos afirmar que el tratamiento que los medios de comunicación dan al concepto de pobreza es un tema recurrente dentro de la academia y cabría citar, dentro de esta línea de trabajo, los estudios desarrollados por Joanna Redden y los métodos empleados en sus investigaciones para llevar a cabo estos análisis (Redden, 2011).

De acuerdo con lo expuesto, los individuos "se preocupan más por la desigualdad de ingresos cuando este se convierte en un problema social" (McCall, 2010, p. 50). Como consecuencia de este fenómeno algunos autores (por ejemplo, Duca y Saving, 2017), se preguntan hasta qué punto la desigualdad conduce a la polarización del debate alrededor de este concepto.

Para concluir con este punto, consideramos que hay otras razones para colocar el concepto de desigualdad de ingresos en el centro del debate público. Una de ellas es la naturaleza del largo plazo inherente a la desigualdad. En este sentido, "la tendencia a largo plazo en la desigualdad salarial ha sido impulsada por dos factores principales: un aumento en extremo superior de los ingresos y la riqueza, $y$, en el extremo inferior, una combinación de reducción en la riqueza y un crecimiento más lento del salario en los buenos tiempos y un descenso del salario durante los malos tiempos" (Fisher y Smeeding, 2016, p. 32). Esto hace que, de acuerdo con Thomas Piketty, "la desigualdad hace que los ciudada- 
nos pierdan su fe en un sistema por el que se sienten injustamente tratados" (2015). En consecuencia, creemos que analizar cómo es la conversación que tienen estos ciudadanos sobre el concepto de desigualdad de ingresos puede aportar ideas más detalladas y específicas sobre su impacto en la sociedad y en los individuos que la integran.

\subsection{Audiencias activas y desigualdad}

La irrupción de los medios digitales y los cambios en el sistema mediático en el que nos encontramos han posibilitado el asentamiento de un escenario donde, cada vez más, nuevas formas de comunicación son posibles, lo que implica que el paso desde el espectador pasivo de los medios tradicionales a un usuario activo que no solo consume contenido, sino que también interacciona con él de diferentes maneras (Soler-Adillón, 2017, p. 2), dando lugar a la formación de una audiencia activa. Tal y como recogen los investigadores Pere Masip, Carlos Ruíz-Caballero y Jaume Suau (2019), el término audiencias activas empezó a utilizarse para distinguir la nueva naturaleza de las audiencias (Bruns y Highfield, 2016), las cuales se comienzan a percibir no solo como activas en la interpretación de textos y mensajes periodísticos, sino también en el sentido de ser productores, de diseminar el contenido y de mantener debates públicos en los entornos en línea (Suau, 2015). En este sentido, los medios digitales juegan un papel central en la creación de nuevas formas de contribuir a la sociedad a través de la interacción entre los usuarios que tiene lugar en las plataformas de redes sociales (Marres, 2017, p. 7). Por ello, este estudio intenta comprender las implicaciones del uso de las redes sociales en el proceso de establecer un tema específico dentro de la agenda. Como sugiere Elena Pavan (2014, p. 441), las redes digitales pueden entenderse como una metáfora y como herramientas empíricas para explorar las relaciones surgidas entre los actores. De ahí que sigamos esta línea propuesta, y que el uso de las redes sociales se entiende en esta propuesta como una herramienta poderosa para producir conocimiento sobre el concepto de desigualdad de ingresos dentro del debate en la esfera pública digital.

Para analizar las plataformas digitales es importante comprender el papel distintivo que desempeña la comunicación en estas redes a menudo densamente entrelazadas de actores, discursos y tecnologías. La madurez democrática de los sistemas políticos parece tener una clara influencia en cómo los votantes y los usuarios (audiencias) interactúan y contrarrestan la información para tratar de cambiar el rumbo de la conversación. Siguiendo a Sanghamitra Bandyopadhyay, "se puede conjeturar que una mayor incidencia de variables de los medios, como periódicos, radios y televisores, y un mayor acceso a las TIC, como un mayor uso de Internet y una mayor penetración de las telecomunicaciones, pueden ser asociadas con niveles más bajos de desigualdad" (Bandyopadhyay, 2014, p. 11). En relación con esto, los medios digitales pueden ser usados como medios alternativos de información y participación, lo cual, teniendo en cuenta el objeto de estudio de este trabajo, cobra relevancia, ya que, como afirma Maria Petrova "los medios de comunicación son la fuente más importante de información sobre políticas públicas para la gente común, y se convierten en una herramienta atractiva que puede ser utilizada por grupos de altos ingresos para influir en la opinión pública [...] por tanto, una mayor desigualdad conduce a un menor nivel de libertad en los medios" (Petrova, 2005, p. 27). Otro aspecto a tener en cuenta serían los prejuicios y la forma en que las audiencias han sido influenciadas por los medios de comunicación, centrándose en el liberalismo y el neoliberalismo (Byrne, 2012; Hall, 2011). Por tanto, el uso de los medios digitales por parte de las audiencias activas supone romper esa barrera de entrada y poner en la esfera pública digital temas o enfoques que, en principio, no son recogidos por los medios de comunicación de masas tradicionales.

De acuerdo con lo expuesto, algunos investigadores también han subrayado la importancia de la comunicación digital y la aparente facilidad de "tener una voz" (lo cual no debe confundirse con tener influencia en otros usuarios) (Díaz-Noci, Pérez-Altable, 2018, p. 89). Con este apunte, tal y como han afirmado Courtois y Verdegem "en la sociedad de la información actual, el acceso y el dominio de los recursos en línea son requisitos previos indispensables para la participación" (2016, p. 1509). En un estudio reciente, han concluido que "el uso de fuentes de apoyo social modera la asociación directa e indirecta entre las fuentes de desigualdad (es decir, la calidad de acceso, las habilidades y la motivación) y la diversidad en los resultados positivos". Además, ambos investigadores recuerdan que "muestra un vínculo aparente entre los recursos fuera de línea y en línea; es una instancia de cómo la inclusión fuera de línea y en línea van de la mano" (ídem). Algunos autores españoles relevantes, como el sociólogo Julio Carabaña (2016), han subrayado el papel de los medios de comunicación para forjar una imagen social de la desigualdad de ingresos.

\section{Metodología}

El objetivo general de este estudio es analizar los patrones de comunicación en redes digitales, específicamente Twitter, del concepto de desigualdad de ingresos (income inequality en inglés). Considerando el objetivo de nuestro estudio, nos formulamos la siguiente pre- 
gunta de investigación: ¿Cómo es la conversación sobre el concepto de desigualdad de ingresos entre los usuarios de plataformas digitales (Twitter)?

Así, para dar respuesta a nuestra pregunta de investigación, realizamos la recogida, y posterior análisis, de los tuits publicados en la plataforma durante el año 2018 completo que contenían las palabras clave "income inequality" en el cuerpo del mensaje. La elección del idioma viene determinada por el hecho de que en inglés nos encontramos con un único concepto, income inequality, mientras que en castellano nos encontramos con diferentes términos: desigualdad económica, desigualdad salarial, desigualdad de ingresos, entre otros. Al tratarse de un estudio previo con un marcado carácter exploratorio, nos parecía más adecuado testear la herramienta con el término inglés porque no da lugar a confusión ya que se trata de un término ampliamente utilizado, establecido y, además, nos permite conformar una muestra lo suficientemente robusta para llevar a cabo el análisis. Esto ha supuesto que los resultados obtenidos se refieran a un contexto anglosajón, con especial prevalencia de tuits con origen Estados Unidos y, menos proporción, Reino Unido.

Los datos se recopilaron utilizando el buscador avanzado de Twitter (Twitter Advanced Search), combinándolo con el programa de scrapping "Data Miner". Finalmente obtuvimos un documento .csv con un total de 102.082 tuits. En este sentido nos gustaría aclarar que, aunque sabemos que el conjunto de datos que provienen directamente desde el buscador no representa la totalidad de tuits publicados, consideramos que el número total de mensajes (102.082) es suficientemente robusto para poder llevar a cabo el análisis y, por tanto, alcanzar los objetivos planteados en este estudio.

Para la elaboración de la muestra, se ha seguido la técnica de la semana construida (Krippendorff, 1990). Esta técnica permite conformar una muestra estratificada por días de la semana. La muestra final se compone de 1.531 tuits. Una vez obtenida la muestra, se llevó a cabo un análisis de contenido de la misma. Nuestro objetivo es realizar un análisis explicativo para encontrar el significado profundo de los mensajes y descubrir cuáles son las inferencias sobre el origen, la naturaleza y los efectos de los mismos. Aunque como hemos explicado anteriormente este trabajo se trata de un análisis exploratorio, nuestro diseño se basa en un enfoque longitudinal y horizontal aplicando repetidamente las mismas técnicas, ya que el objetivo final del proyecto es obtener un análisis sistemático de varios años de opinión pública en Twitter sobre el concepto de desigualdad de ingresos. Basándonos en la revisión de la literatura y en trabajos previos llevados a cabo por miembros del grupo de investigación (Odriozola; Pérez-Arozamena y Fernández-Planells, 2018; Pérez-Altable et al, 2020) hemos definido una serie de categorías para el análisis, que son las siguientes:

\section{Categorías contextuales}

1.1. Id: número identificativo del tuit

1.2. Nombre de codificadora: persona encargada de codificar el tuit

1.3. Fecha de codificación: fecha en que se realizó la codificación

1.4. Fecha de publicación: fecha en que fue publicado el tuit

1.5. URL del mensaje: enlace para recuperar el tuit

1.6. Nombre de usuario: autor del tuit

\section{Contenido del tuit}

2.1. Texto: texto completo del tuit

2.2. Nombres mencionados: nombres que se citan o interpelan en el tuit

2.3. Hashtags: etiquetas utilizadas dentro del tuit

2.4. Cuentas mencionadas: otros usuarios que se citan o interpelan en el tuit

2.5. Enlaces, contenido multimedia: si el tuit incluye enlace o contenido multimedia

2.6. Tipo de contenido multimedia: se especifica el tipo de contenido multimedia que incluye el tuit

2.6.1. Imagen

2.6.2. Vídeos

2.6.3. Gráficos

2.6.4. Otros

\section{Income inequality en Twitter}

3.1. Carácter de la desigualdad

3.1.1. Micro: la desigualdad mencionada en el tuit hace referencia a diferencias entre personas dentro de una comunidad

3.1.2. Macro: la desigualdad mencionada en el tuit hace referencia a diferencias entre comunidades (ciudades, regiones, países...)

3.2. Tema principal del tuit

3.2.1. Healthcare: la desigualdad hace referencia a temas de salud

3.2.2. Education: la desigualdad hace referencia a temas de educación

3.2.3. Environment and climate: la desigualdad hace referencia a temas de medioambiente

3.2.4. Poverty: la desigualdad hace referencia a la pobreza 
3.2.5. Unemployment rates: la desigualdad hace referencia a la problemática del desempleo

3.2.6. Social classes: la desigualdad hace referencia a las diferencias entre clases sociales

3.2.7. Public safety: la desigualdad hace referencia a la seguridad pública

3.2.8. Salaries: la desigualdad hace referencia a salarios

3.2.9. Gender inequality: la desigualdad hace referencia al impacto del género

3.2.10. Migration: la desigualdad hace referencia a la migración o a la condición de migrante

3.2.11. Tax system: la desigualdad hace referencia al sistema de impuestos

3.2.12. Commercial balance: la desigualdad hace referencia a temas comerciales

3.2.13. Other: otro tipo de desigualdades que ni hayan sido consideradas en el análisis

3.3. Ámbito geográfico

3.3.1. Local: el tuit se refiere a un pueblo o ciudad concreto

3.3.2. Regional: el tuit se refiere a una región específica

3.3.3. Estatal: el tuit se refiere a un estado concreto

3.3.4. Internacional: el tuit se refiere a dos o más países

\subsubsection{No aplica}

En este sentido cabe señalar que las categorías han sido a lo largo de todo el proceso de análisis siguiendo una aproximación deductiva. Tres investigadoras se encargaron de codificar todos los tuits y de generar un diario de campo que permita, en futuros trabajos, refinar la herramienta de análisis. Este proceder está relacionado con un enfoque de teoría fundamentada o Grounded Theory, basado en un análisis sistemático de datos, una recopilación y análisis de datos simultáneos (el diseño longitudinal del estudio de investigación nos permite proceder así), siguiendo un diseño interactivo hasta la saturación teórica, es decir, hasta encontrar las categorías centrales que nos permiten explicar cómo la opinión pública evoluciona y se construye utilizando redes sociales digitales, como Twitter, en torno a cuestiones públicas centrales de nuestras sociedades, como la que estamos tratando.

\section{Resultados}

Debido a la gran cantidad y diversidad de variables analizadas y dado el límite de espacio con el que contamos, en este apartado nos centraremos en explicar los resul- tados obtenidos que consideramos más relevantes para este estudio.

En primer lugar, destacamos que solo en un 4,49\% de los mensajes se nombra a alguien de manera explícita. Dentro de ese porcentaje, encontramos que son los actores políticos los más mencionados. De este modo, Donald Trump aparece en un 17\% de los mensajes, seguido de los líderes demócratas Bernie Sanders $(16,17 \%)$ y el anterior presidente de los Estados Unidos, Barack Obama (14,7\%).

En cuanto a los enlaces incluidos en los mensajes, un $43 \%$ de los mensajes publicados por los usuarios incluyen enlaces a otros sitios web, mientras el 13\% de esos enlaces corresponde con contenido multimedia. En este sentido, en la figura 1 podemos observar que dentro del contenido multimedia lo más compartido son imágenes, seguido de gráficos y vídeos. "Otros" hace referencia a contenido multimedia como GIFS que no se tuvieron en cuenta al establecer las variables iniciales pero que, tal y como hemos expresado anteriormente, ya que se trata de un estudio exploratorio se incluirán en próximos análisis dada su creciente influencia y uso por parte de las audiencias activas.

En cuanto a los enlaces compartidos que no son mul-

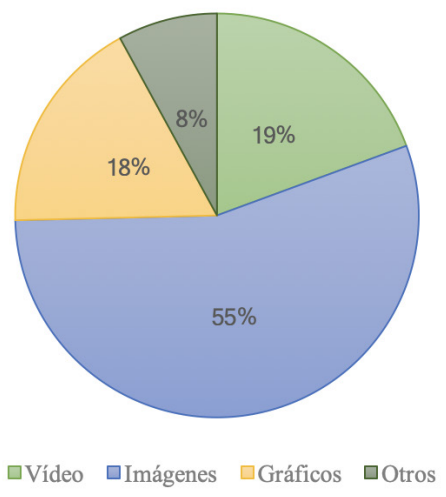

Figura 1. Tipo de contenido multimedia. Elaboración propia.

timedia, nos encontramos fundamentalmente con dos tipos (figura 2). Por un lado, los enlaces a noticias publicadas en medios de comunicación y, por otro, enlaces a artículos e informes académicos publicados sobre la desigualdad de ingresos. En un trabajo previo realizado por miembros del grupo que analizó las fuentes (Pérez-Altable, Fernández-Planells y Serrano-Tellería, 2019) nos encontramos con que el uso de fuentes académicas estaba muy presente, por lo que el hallazgo de las mismas en este estudio confirma esta tendencia en 


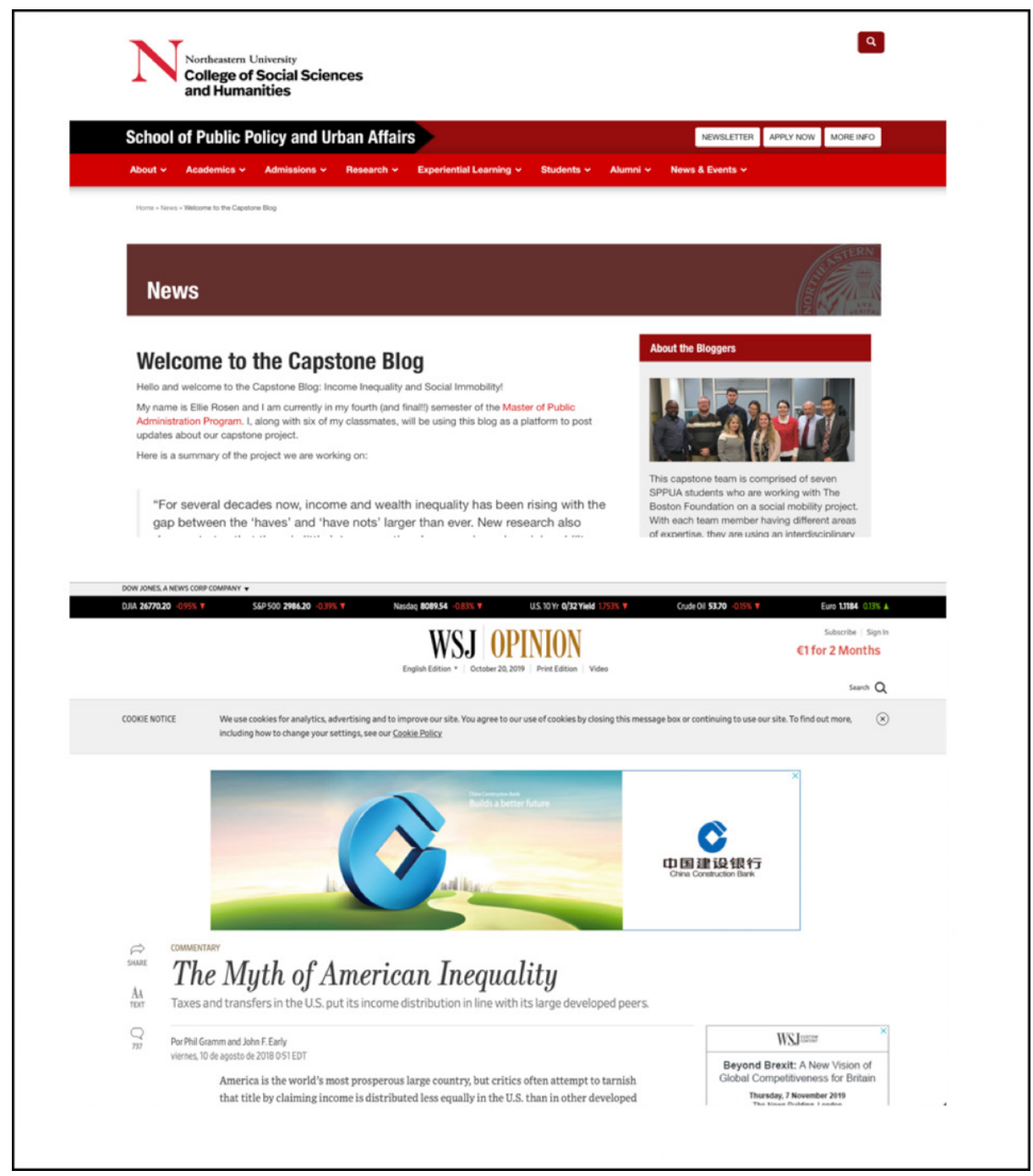

Figura 2. Contenido compartido por los usuarios. Elaboración propia

su uso y presencia al tratar este tipo de temas.

En cuanto al carácter de la desigualdad, esto es, si el mensaje se refiere a un nivel micro de desigualdad (diferencias entre personas dentro de una misma comunidad: país, región, ciudad...) o a un nivel macro (diferencias entre comunidades) nos encontramos que un $44,89 \%$ de los mensajes hacen referencia al nivel micro, mientras que en un $23,5 \%$ se refieren a desigualdades a nivel macro. Cabe destacar que hay un 32,61\% de los mensajes a los que no se aplica esta clasificación, pues no hacen referencia si de lo que se publica se refiere a una desigualdad a nivel micro o macro y el contenido del tuit es escaso para deducir correctamente su carácter. Por otro lado, dentro del ámbito geográfico, tal y como podemos observar en la figura 3, nos encontramos que la mayoría de mensajes se refieren al ámbito estatal, seguido por el internacional y el local.

Por último, en cuanto al tema principal de los mensajes nos encontramos con una evolución de las categorías establecidas inicialmente. Como hemos explicado ante- riormente, las categorías de análisis se establecieron en base a términos y conceptos basados en la literatura académica sobre el tema y en trabajos previos del grupo de investigación. Lo que nuestro estudio nos muestra es una evolución de la conversación, y esto se refleja con el incremento de la frecuencia en la que "Otros" aparece como tema principal del mensaje. Ya que se trata de un trabajo exploratorio y en ningún momento es nuestra

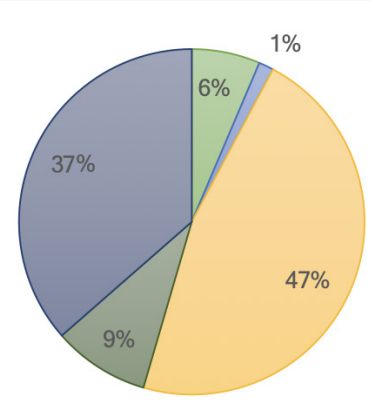

$\square$ Local $\square$ Regional $\square$ Estatal $\square$ Internacional $\square$ No aplica

Figura 3. Ámbito geográfico. Elaboración propia. 


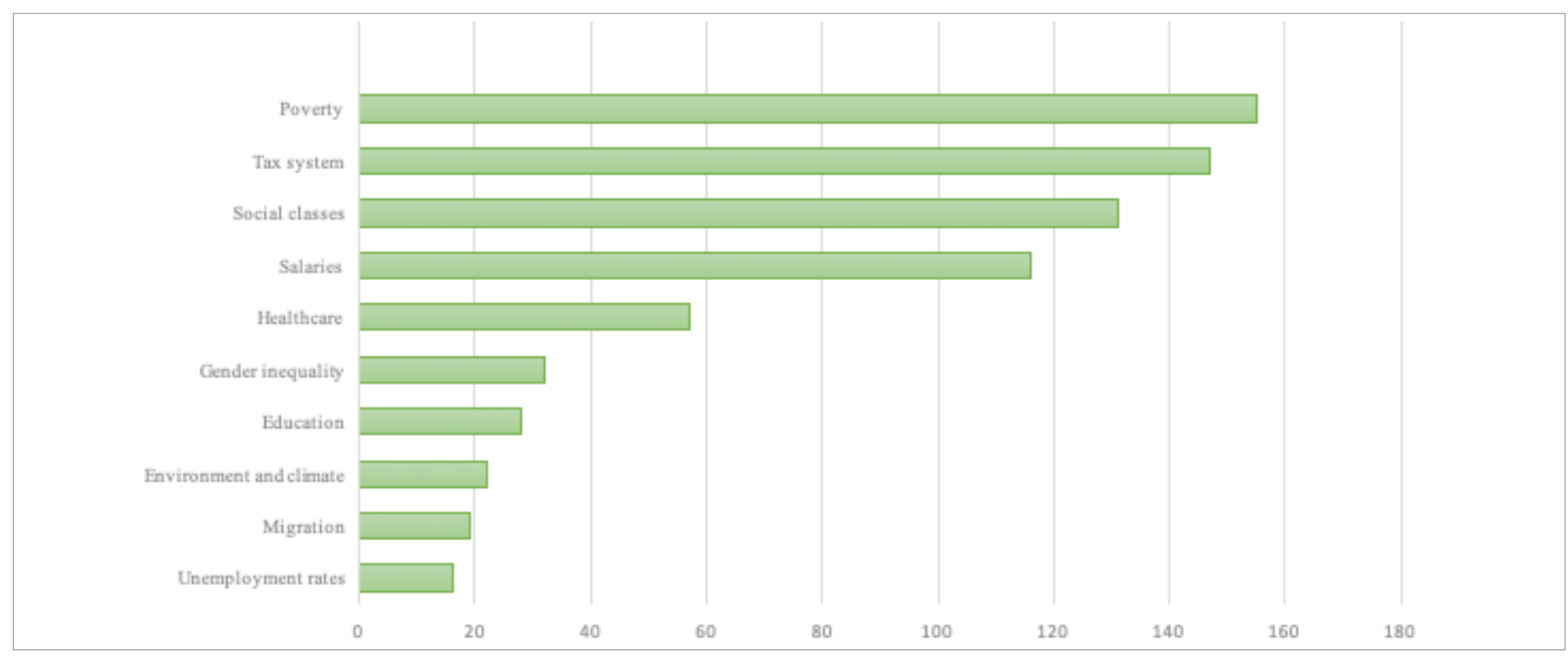

Figura 4. Temas principales de los mensajes. Elaboración propia.

intención mostrar estos resultados como concluyentes, porque deberán ser ampliados en futuras investigaciones, consideramos que este resultado forma parte del proceso y es parte de la evolución del estudio.

Aclarado este punto, como hemos señalado, nos centraremos en las categorías que aparecen como "Tema principal del mensaje" descartando la categoría "Otros". En el siguiente gráfico se observa cómo se distribuyen los temas en relación a los mensajes publicados por los usuarios, ordenados de mayor a menor frecuencia (figura 4).

En primer lugar, observamos que el tema que más aparece en relación con la desigualdad de ingresos es el de pobreza. Aunque en un principio pudiera parecer que desigualdad de ingresos y pobreza es lo mismo no es así, la pobreza atiende a múltiples factores y, en relación a la desigualdad de ingresos, es una consecuencia de este tipo de desigualdad. Algunos ejemplos de este tipo de mensajes son:

@Ntl_homeless, 5:30pm, Aug 10, 2018, "Income inequality and wealth disparity have increased under Republicans and Democrats over the past four decades. Poor people and moral leaders have been calling for a Poor People's Campaign for a long time."

@niishthebish, 7:34am, Nov 11, 2018, "40 million Americans are poor. 73 million Americans (and growing) are enrolled in Medicaid and CHIP. Our income inequality Gini index is higher than most other developed countries. But tell me again it isn't "some specter haunting American life" ?"

@Tajalithaca, 5:19am, Oct 24, 2018, "Have you forgotten the STRESS that accompanies poverty? Money erodes empathy - fact. You were okay being "well-off" while the majority of your "countrymen" struggled. You were "smart" and "getting ahead". Do you love income inequality?"

El segundo tema que aparece con más presencia es el de sistema de impuestos. En este sentido, debido al uso de las palabras clave income inequality en inglés para conformar la muestra, muchos de los mensajes hacen referencia al sistema de impuestos de Estados Unidos y cómo afecta a la desigualdad de ingresos dentro del contexto americano. Detrás de los mensajes que hacen referencia al sistema de impuestos, se encuentra el debate de si un sistema de impuestos más progresivos reduciría la desigualdad de ingresos entre la población. Por ejemplo:

@FNC_Ladies_Rule, 2:13am, Oct 24, 2018, "So in your opinion is okay that we have a huge deficit and income inequality because the wealthy pay more in taxes? That is some seriously messed up economic theory."

@Pairsonnalities, 6:14am, Feb 1, 2018, "Stigmabase I NAM - Trump is still trying to sell tax cuts for the wealthy to Middle America - The growing level of income inequality is a cancer that threatens the nation. In the Democratic response, Rep. Joseph 
Kennedy III of Massachusetts criticized... http://dlvr. it/Q⿱Dfmch"

@iwelsh, 5:05pm, Aug 10, 2018, "If you have a society with high wealth and income inequality (most societies today, and especially true in India), you should have targeted consumption taxes at stuff you genuinely want consumed less, and high marginal tax rates on the rich."

Otro de los temas que tienen bastante presencia en la conversación en torno al concepto de desigualdad de ingresos en Twitter es el de clases sociales. Esto se debe a que toda desigualdad está fuertemente relacionada con la clase social e incluso, como es el caso de la desigualdad de ingresos, determina la pertenencia a una determinada clase social u otra. Teniendo en cuenta esto, parece bastante lógico que la clase social como tema central esté presente en los mensajes difundidos en Twitter sobre la desigualdad de ingresos. Algunos ejemplos de mensajes que tratan la clase social son:

@wordportrait_10, 1:57am, Oct 24, 2018, "Used to be $33 \%$ of $r$ nation revenue came for corps... now it's under 9\%... they use more of all the services we all pay for... and CT is \#2 in income inequality... this is what happened in 1930... r middle class is fading away bc lions share is going to the top!"

@adwrein, 10:52pm, Sep 24, 2018, "I find my working classness helps with clients, meet far more working class clients than not given statistics around income inequality and mental health problems not surprising. It's always been other professionals that have had more of an issue with working class psychs."

@IronStache, 1:21am, Sep 24, 2018. "Wisconsin has a good opportunity to elect someone who understands working people. Income inequality in America is at levels even higher than those in ancient Rome, according to a recent study from two historians, Walter Schiedel and Steven Friesen,"

La desigualdad de ingresos está relacionada con la distribución de la renta, tanto la renta capital como la rentas obtenidos por el trabajo (salario). Por tanto, es lógico pensar que el salario sea uno de los temas principal en los mensajes que tratan la desigualdad de ingresos, tal y como muestran los siguientes mensajes:

@asapna, 6:07pm, Oct 24, 2018, "brilliant story comparing how workers at Amazon are paid today vs. Sears in its heyday $\&$ what that says about corporate America + income inequality https:// nytimes.com/2018/10/23/business/economy/amazon-workers-sears-bankruptcy-filing.html... by $₫$ NelsonSchwartz + amcorkery5"

amikegoodma, 1:48pm, Oct 24, 2018, "Years ago Sears put $10 \%$ pretax $\$$ into FTE retirement plan. Now, shareholders are supreme. \$AMZN recently bumped up min wage to $\$ 15 / \mathrm{hr}$ but also nixed its generous worker stock ownership plan. \#NYT says this demotion of workers is tied to income inequality."

@mythousandfaces, 7:17am, Sep 24, 2018, "The median annual salary in Chile is under a thousand dollars, theress insane income inequality, and a huge swathe of the population is still traumatized by decades of brutal rule. also Pinochetss Chile did worse than Maduross Venezuela and needed constant foreign bailout."

Por último, cabe señalar que, tal y como se puede observar en el Gráfico 3, aparecen una serie de temas en menor proporción, pero que igualmente son importantes como, por ejemplo, la sanidad, la educación o la desigualdad de género:

@Supertitionsee, 1:02am, Sep 24, 2018, "I liked Bernie, I liked his message about healthcare and income inequality, but for the good of us all, I hope he chooses not to run, but to support someone we can all get behind. The last thing we need is another divisive candidate."

aChessmanmark, 4:29am, Oct 24, 2018, "The Trump Presidency is a direct consequence of the dumbing down of America. Supporting and financing education is a very low priority in this country. It also correlates to income inequality. public school are under funded."

@AyeshaRekhi, 9:50pm, Feb 1, 2018, “Happy to host discussion with allOAsiaPacific this morning on achieving \#equalpay for work of equal value. Important takeaway - reducing the gender pay gap is key to tackling growing income inequality. \#SDGs \#gamechanger"

\section{Conclusiones}

Como hemos explicado anteriormente, estos resultados y conclusiones han permitido diseñar una herramienta de análisis de la conversación en Twitter sobre el con- 
cepto de desigualdad de ingresos (income inequality) así como extraer unos primeros resultados y conclusiones preliminares que se ciñen exclusivamente al contexto anglosajón y al año 2018. Así, por ejemplo, las fuentes utilizadas (medios, por ejemplo) o las personas citadas (políticos como Donald Trump, Bernie Sanders, etc.) o la temáticas que centran el debate en Twitter hacen clara referencia al contexto americano del 2018 del que provienen gran parte de los tuits de la muestra debido a la decisión metodológica de utilizar "income inequality" como palabras clave de búsqueda en Twitter. Por ello, hay que tomarlos con cautela y,no son generalizables ni extrapolables.

Este estudio sienta las bases para futuras investigaciones y muestra las tendencias en un tema de máxima actualidad como es la desigualdad. Así, analizando nuestros resultados podemos observar como hay una prevalencia de los mensajes a nivel micro sobre los marcos o la prevalencia de temas como la pobreza, el sistema fiscal, las clases sociales o los salarios (tan interrelacionados entre sí a su vez) sobre el resto de temas.

Lo que este estudio exploratorio muestra es que la conversación que se establece en la esfera pública digital en relación al concepto de desigualdad de ingresos (income inequality) es muy dinámica y evoluciona con rapidez, ya que la lista de términos y temas relacionados se ha modificado considerablemente respecto a estudios anteriores como podemos observar en el incremento de mensajes cuyo tema principal pertenece a la categoría "Otros". Por ejemplo, de nuestro análisis se desprende que se podrían incluir temas principales como la vivienda, la desigualdad racial o la calidad de vida, entre otras. Por tanto, el principal resultado es la necesidad de ampliar los temas incluidos en la herramienta de análisis para dar cabida a los nuevos temas que han surgido en relación al concepto de desigualdad de ingresos (income inequality).

También consideramos que, de cara a futuras investigaciones, podría ser relevante analizar el tono del mensaje, es decir, si se trata de un mensaje positivo, negativo o neutro. Creemos que este análisis puede aportar información que complementaria de una manera muy positiva en análisis que presentamos en este estudio.

En cualquier caso, como venimos diciendo a lo largo del documento, este estudio sienta las bases de cara a futuras investigaciones, ya que hemos obtenido información muy valiosa para su futuro diseño $y$, por eso mismo, no ha de ser entendido como el final de la investigación, si no como parte del proceso de la misma, siguiendo las premisas de la Grounded Theory que ha guiado la propuesta metodológica de este estudio. Hemos realizado un test de la metodología en profundidad que nos permitirá adaptar los temas a su evolución, confirmar resultados de estudios previos y describir la tendencia de los temas.

\section{Nota}

Este artículo pertenece al proyecto de investigación"News, Networks and Users in the Hybrid Media System (Newsnet). Transformation of News and Media Industry in the Post-Industrial Era", referencia: RTI2018095775-B-C43, financiado por el Ministerio de Ciencia, Innovación y Universidades - Gobierno de España.

\section{Agradecimiento}

Nos gustaría agradecer especialmente la labor de los revisores de este artículo por sus comentarios y sugerencias que han contribuido a mejorarlo notablemente.

\section{Referencias}

Aghion, P.; Akcigit, U; Bergeaud, A.; Blundell, R. y Hemous, D. (2019). Innovation and Top Income Inequality. The Review of Economic Studies, 86(1), p.1-45.

Bandyopadhyay, S. (2014). Are Mass Media and ICTs Associated with Inequality and Poverty?. Economic Well-Being and Inequality: Papers from the Fifth ECINEQ Meeting (Research on Economic Inequality, (22), p. 185-216.

Bruns, A. y Highfield, T. (2016). Is Habermas on Twitter? Social media and the public sphere. En: The Routledge companion to social media and politics (pp. 56-73). New York: Routledge.

Byrne, R. J. (2012). Framing Income Inequality in the media: Is There a Liberal or Neoliberal Bias (Tesis de máster).Georgia Southern University. Recuperado de: https://digitalcommons.georgiasouthern.edu/ cgi/viewcontent.cgi?article=1622\&; context=etd

Carabaña, J. (2016). Ricos y pobres. Madrid: Libros de la Catarata.

Courtois, C.; Verdegem, P. (2016). With a little help from my friends: An analysis of the role of social support in digital inequalities. New Media \& Society 18(8), p.1508-1527.

Colleoni, E., Rozza, A. y Arvidson, A. (2014). Echo chamber or public sphere? Predicting political orientation and measuring political homophily in Twitter using big data. Journal of communication, 64(2), p. 317-332.

Díaz-Noci, J. y Pérez-Altable, L. (2018). Dealing with the crisis: Income inequality and digital media in the Basque Country (2008-2018). En: Actas del $X$ Congreso Internacional de Ciberperiodismo. Profesionales y audiencias en el ecosistema móvil (pp. 86-102). Leioa: Servicio Editorial de la UPV/EHU,

Duca, J. V.; Saving, J. L. (2017). Income Inequality, Media Fragmentation, and Increased Political Polarization. Contemporary Economic Policy, 35(2), p. 392-413.

Fisher, J.; Smeeding, T. M. (2016). Income Inequality. En: The Poverty and Inequality Report 2016 (pp. 32-38). Stanford: The Stanford Center 
on Poverty and Inequality.

Hall, S. (2011). The Neo-liberal Revolution. Cultural Studies, 25(6), p. 705-728.

Keeley, B. (2018). Desigualdad de ingresos. La brecha entre ricos y pobres. París: Esenciales OCDE, OECD Publishing. Recuperado de: https://www.oecd-ilibrary.org/docserver/9789264300521-es. pdf?expires $=1586959844 \&$ tid=id\&accname=guest\&checksum $=-$ 183509C2EAA9FDA23F54AF3CDD8F4AE9

Loader, M. y Mercea, D. (2011). Networking Democracy? Social media innovations and participatory politics. Information, Communication $\theta$ Society, 14(6), p. 757-769.

Mccall, L. (2010). American Beliefs about Income Inequality. Chicago: Department of Sociology, Northwestern University.

Mckay, A. (2002). Defining and Measuring Inequality. Inequality Briefing, 1. London: UK Department for International Development (DFID) by the Economists' Resource Centre (ERC).

Marres, N. (2017). Digital Sociology. Cambridge: Polity Press.

Masip, P.; Ruiz-Caballero, C. y Suau, J. (2019). Active audiences and social discussion on the digital public sphere. Review article, El Profesional de la Información, 28(2), e280204.

Odriozola, J.; Pérez-Arozamena, R. y Fernández-Planells, A. (2019). El papel de los Legacy media en la construcción de la opinión pública: conceptualización, enfoques y participación en el tratamiento de la desigualdad económica en seis cibermedios española. En: Actas del XXV Congreso Internacional de la Sociedad Española de Periodística. Oportunidades y riesgos del periodismo hiperconectado. Leioa: Servicio Editorial de la UPV/EHU.

Pavan, E. (2014). Embedding Digital Communication Within Collective Action Networks: A Multidimensional Network Approach. Mobilization, 19(4), p.441-455.

Pérez-Altable, L., Fernández-Planells, A. y Serrano-Tellería, A. (2019). Analyzing the network of connective media around the concept of income inequality on Twitter. Comunicación presentada en Annual Conference of the International Association for Media and Communication Research, IAMCR, 7-11 de julio de 2019, Facultad de Ciencias de la Información, Universidad Complutense de Madrid.

Pérez-Altable, L; Pérez-Arozamena, R.; Linares-Lanzman, J.; Odriozola-Chené, J; Fernández-Planells, A.; Serrano-Tellería, A. y Díaz-Noci, J. (2020). The Crisis, the People and the Media: How Digital Public
Opinion Debates on Income Inequality. En: S. Peña Fernández, K. Meso Ayerdi y A. Larrondo Ureta (Eds.), Active Audiences. Empowering Citizens' Discourse in the Hybrid Media System, (pp. 131-143). Madrid: McGraw Hill.

Petrova, M. (2005). Inequality and Media Capture. Cambridge, MA: Harvard University.

Pickett, K.E; Wilkinson, R.G. (2015). Recalibrating Rambotti. Disentangling concepts of poverty and inequality. Social Science and Medicine, (139), p. 132-134.

Picketty, T. (11 de enero de 2015). La mayor desigualdad la provoca el desempleo. El País. Recuperado de: https://elpais.com/ economia/2015/01/09/actualidad/1420836043_696183.html

Redden, J. (2011). Poverty in the News. A framing analysis of coverage in Canada and the UK. Information, Communication \& Society, 14( 6), p. 820-849.

Savage, M. (2016). Are we seeing a new 'inequality paradigm' in social science?. London School of Economics (web). Recuperado de: https:// blogs.lse.ac.uk/politicsandpolicy/are-we-seeing-a-new-inequality-paradigm-in-social-science

Soler-Adillón, J. (2017). Tres conceptos clave en medios digitales. Hipertext.net, (15), p. 1-3. DOI: 10.2436/20.8050.01.38

Song, Jae; Price, D. J.; Guvenen, F.; Bloom; N.; von Wachter, T. (2019). "Firming up inequality".

The Quarterly Journal of Economics, 134(1), 1-50.

Suau, J. (2015). Citizens and online media participation. Attitudes and motivations towards participatory journalism and other online practices in London and Barcelona (Tesis Doctoral). Universitat Ramon Llull. Barcelona.

CV

Laura Pérez-Altable. Es Doctora en Comunicación Social por la Universitat Pompeu Fabra (2016) con una tesis sobre movimientos sociales y análisis de redes. Profesora asociada en la Universitat Pompeu Fabra y Universidad Nebrija, es miembro del grupo de investigación DigiDoc (Departamento de Comunicación, Universitat Pompeu Fabra). Ha realizado una estan-

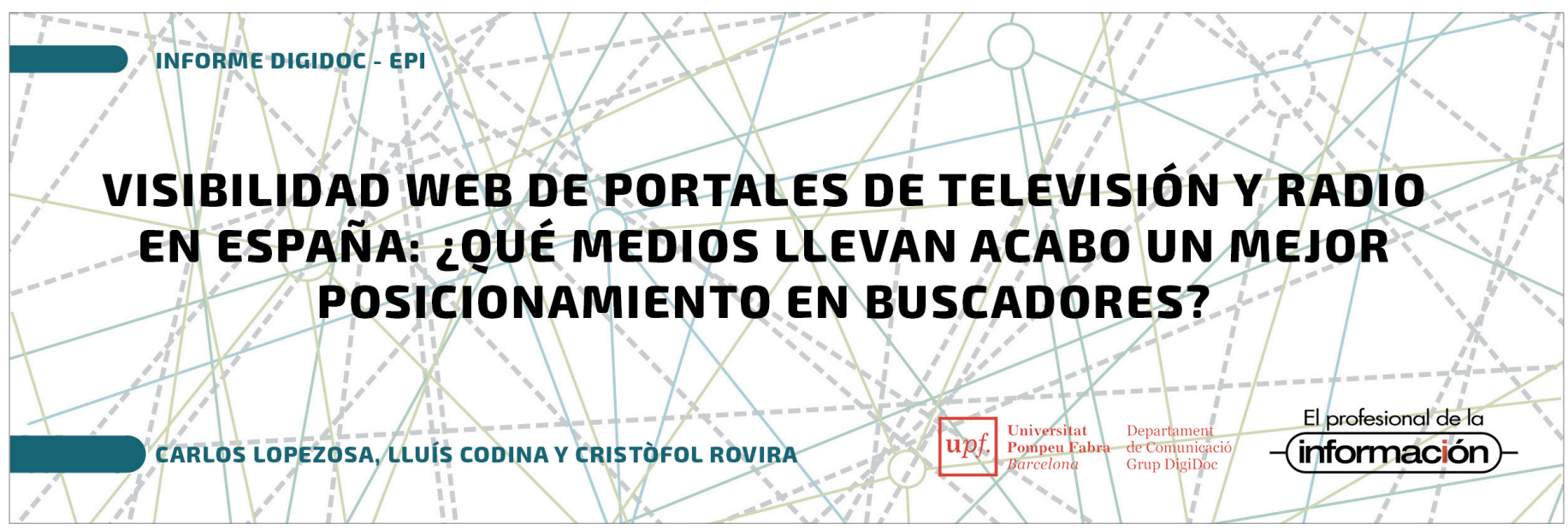


cia de investigación en el Departamento de Sociología de la Universidad de Manchester. En los últimos años ha participado en diferentes proyectos competitivos financiados del Plan Nacional I+D sobre audiencias activas, periodismo y esfera pública digital. Sus principales líneas de investigación son el estudio de los movimientos sociales y la participación ciudadana, así como el análisis de la esfera pública digital a través del análisis de redes sociales, tanto cualitativa como cuantitativamente. https://upf.academia.edu/LauraAltable

Ana Serrano Tellería. Es profesora Contratada Doctora Interina, pertenece como investigadora posdoctoral a los siguientes centros: Media Studies Department, Universiteit van Amsterdam; OsloMet Digital Journalism, Oslo Metropolitan University; DIGIDOC, Universidad Pompeu Fabra; LabCom.IFP, Universidad de Beira Interior, Portugal; MESO, Universidad de San Andrés, Argentina, y Northwestern University, EEUU; Innovation in Digital Media, Universidad Carlos III e Innovamedianet: A network of researchers. Desde que obtuviera el 'Premio Extraordinario de Doctorado' en 2012 por su tesis (2010) "Diseño de nodos iniciales en cibermedios: un estudio comparativo", su labor académica y profesional se ha centrado en el estudio de medios y su alfabetización; ecología de medios; diseño de interfaces y comunicación cros/multi/transmedia. Con más de 90 publicaciones, participación en proyectos y miembro de laboratorios de investigación e innovación nacionales e internacionales; ha ejercido como periodista en medios y gabinetes de comunicación; consultora de medios; responsable de proyectos de investigación; actriz, cantante y ayudante de dirección; manager de artistas; gestora de proyectos culturales y cooperación así como organizadora de eventos. Ha conseguido relevantes becas de la Unión Europea, Fundación Botín, Gobierno de España, Gobierno de Cantabria, Gobierno de Brasil, etc. Ha sido coordinadora de prácticas externas, 10 y 3 curso (actualmente) del Grado de en Periodismo. https://uclm.academia. edu/AnaSerranoTellería
Ariadna Fernández-Planells. Es doctora en Comunicación Pública por la Universidad Pompeu Fabra de Barcelona. Actualmente combina la docencia con la investigación en el Departamento de Comunicación Audiovisual, Documentació e Historia del Arte en la Universidad Politécnica de Valencia. El área de especialización de esta investigadora es la de los nuevos medios. $\mathrm{Ha}$ trabajado en numerosas investigaciones relacionadas con el uso de los medios sociales, del activismo y los nuevos hábitos informativos de la juventud. Su búsqueda siempre versa alrededor del impacto de las TIC en diferentes ámbitos de la vida. Ha tenido varias estancias en universidades internacionales, una de las más recientes en la universidad de Cardiff, una de las más destacadas en investigación en Comunicación Social. En los últimos cinco años ha participado en diferentes proyectos de investigación competitivos financiados del Plan Nacional de I + D sobre audiencias activas y periodismo y a nivel europeo sobre jóvenes, bandas urbanas y uso de las redes. Es miembro del Grupo de Investigación en Juventud, Sociedad y Comunicación (JOVIScom) de la Universidad Pompeu Fabra y del grupo Trademetrics de la UPV. https://upv-es.academia.edu/AriadnaFernandezPlanells

El Observatorio de Cibermedios es una producción del Grupo de Investigación en Documentación Digital y Comunicación Interactiva (DigiDoc) del Departamento de Comunicación de la Universitat Pompeu Fabra.

El Observatorio de Cibermedios (OCM) forma parte del proyecto del Plan Nacional "Creación y contenido interactivo en la comunicación de información audiovisual: audiencias, diseño, sistemas y formatos". CSO2015-64955-C4-2-R (MINECO/ FEDER), Ministerio de Economía y Competitividad (España). 